\title{
THE FORMATION OF PULMONARY HYALINE MEMBRANES IN THE NEWBORN BABY
}

BY

\author{
T. WADE-EVANS \\ From the Department of Pathology, Dudley Road Hospital, Birmingham
}

(RECEIVED FOR PUBLICATION APRIL 2, 1962)

\begin{abstract}
Although many attempts have been made to explain the formation of the pulmonary hyaline membranes found in newborn babies, their exact pathogenesis remains unknown. A number of theories of origin by aspiration stemmed from the description of membranous structures in the lungs of babies who had also aspirated vernix caseosa (Hochheim, 1903); however, most workers have abandoned these in favour of origin within the lung itself, either from the lining epithelium, or from material, particularly protein, lost through the pulmonary capillaries, and this last view seems most likely to be correct. Unfortunately, it was for long impossible to show the presence of fibrin, and the origin of the membranes from serum protein alone was difficult to accept; such proteins would form membranes only if they were grossly altered and made insoluble. For this reason the demonstration of the fibrin content of the membranes by Gitlin and Craig (1956), who used fluorescent antibodies, was of the greatest importance. Their work has received partial confirmation from the electron microscopic study of van Breemen, Neustein and Bruns (1957), who found material with the branching pattern and striation characteristic of fibrin in membranes produced by oxygen poisoning in the guinea-pig, although these were shown less convincingly in membranes from babies' lungs; Aronson (1961) found that the membranes reacted as would fibrin to enzymatic digestion.

In the investigation reported here, orthodox histological methods were used to show the presence of fibrin in developing hyaline membranes, and were applied to a large series of cases to allow the formation and fate of the membranes to be followed in greater detail.
\end{abstract}

\section{MATERIAL AND METHODS}

The observations presented here were made on material from babies who had died in the neonatal period at St. Mary's Hospital, Manchester, during the 10-year period, 1949-1958. Of 800 babies examined at autopsy, sections of the lungs were available in 791 , and of those $143(18 \cdot 1 \%)$ contained hyaline membranes of the type associated with respiratory distress; membranous structures were seen in a further 32 cases, but, as has been reported elsewhere (Wade-Evans, 1961a), these were considered to be the result of pneumonia.

In this study sections were examined after staining with haematoxylin and eosin, and by a variety of other methods, greatest importance being attached to the use of Mallory's phosphotungstic acid haematoxylin (P.T.A.H.); as early workers, using similar routine methods, had been unable to demonstrate the presence of fibrin in hyaline membranes, the application of this method is considered in some detail.

The histological demonstration of fibrin is often difficult; in routine practice, the material is identified in part by the strong eosinophilia which results from its high content of basic amino acids, and in part by its structure. Additional evidence is given by the use of the so-called 'fibrin stains', including P.T.A.H. All of those, however, have disadvantages: they may fail to stain fibrin itself when, for example, this is present in compact old thrombus; and none is specific for fibrin, other substances, including the squames found in amniotic fluid or in the lungs of many newborn babies, being also stained. The action of P.T.A.H. in staining fibrin has been investigated by Gitlin and Craig (1957), using sections of Zenker-fixed gels made from purified fibrinogen and thrombin; these workers found that the characteristic deep blue staining, referred to here as 'positive' staining, occurred only in the presence of other substances, in particular of albumin and glutathione. They concluded that these substances had an action, similar to that of the serum secondary coupling factor of Laki and Lorand (Lorand, 1950), in promoting cross-linkages between strands of fibrin, and that in the absence of crosslinkages positive staining did not occur. Preliminary observations made here on the staining of fibrin 
derived from whole plasma were not, however, in complete accord with this view. Thin films were made by spreading drops of platelet-rich plasma on clean microscope slides; clotting followed clumping of the platelets which formed small masses adherent to the glass. The structure of the films depended upon the temperature of clotting; at $37^{\circ} \mathrm{C}$. the fibrin formed strands visible with the light microscope, and these were coarsest where they radiated from the platelet clumps; at room temperature or at $4^{\circ} \mathrm{C}$., it usually formed a structureless gel. The staining of such films with P.T.A.H. depended not only on their structure but on the fixative used; with formalin, the visible strands stained deep blue, but not the structureless gels; with Zenker's fluid (a coagulant fixative) while the strands still stained intensely, the structureless gels now also showed local areas of uniform positive staining; these areas were not present, whatever the fixative, if the gels had been washed in saline before fixation, and they appeared to be due to included serum proteins. Thus, it will be seen that fibrin gels could be prepared from plasma in a form which did not stain with P.T.A.H., although the concentration of albumin present was much greater than Gitlin and Craig thought necessary for positive staining; it is likely that the staining of the strands depends on their physical form occurring when they are sufficiently coarse, and that the material stained may well be fibrin itself.

In considering the problems raised by hyaline membrane formation, two important considerations must be remembered in interpreting sections stained by P.T.A.H. First, positive staining is not in itself enough to identify a substance as fibrin; additional evidence of identity is necessary, and from what has been said, this is best given by the material's structure, when this shows characteristic thin branching strands. Second, fibrin, even that derived from plasma, may be present in the form of a gel, or of the film produced by the syneresis of such a gel, without positive staining.

\section{OBSERVATIONS}

\section{The Early Stage of Membrane Formation}

Although the membranes seen in the lungs of the newborn are commonly called 'hyaline', few are truly hyaline in the sense of being structureless, transparent or 'glassy'. They are formed of granular or fibrillary eosinophil material with various inclusions, of which squames and pyknotic or fragmented nuclei are the most common; some are of relatively open texture, some contain coarse strands or appear laminated, and a few are more dense and homogeneous. When stained with

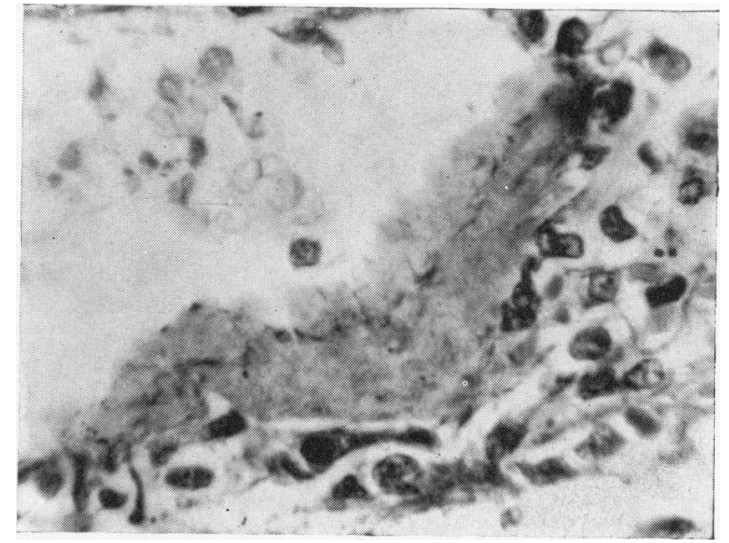

Fig. 1.-Hyaline membrane from the lung of a 23-hour-old baby, showing a fine positively staining strand.

(Phosphotungstic-acid haematoxylin (P.T.A.H.) $\times$ 620.)

P.T.A.H. many are seen to contain fine positively staining strands (Fig. 1) which may be single or branching and which vary in number and length; these are often surrounded by granular material and they resemble the strands in a formalin-fixed plasma clot. It is difficult to identify such strands with certainty on this appearance alone so that their presence in itself gives little helpful information.

Fortunately, in a number of early cases, such as those described below, it is possible to trace the origin of the membranes from fluid containing material that can be identified as fibrin with greater certainty.

Case 1. The pregnancy of a 25-year-old, gravida 2, was complicated by pre-eclamptic toxaemia. Labour commenced spontaneously at 28 weeks, and was rapid, with normal delivery of a female child weighing $0.62 \mathrm{~kg}$. The child was cyanosed at birth, respiration being established slowly and never becoming regular, and died at $3 \frac{1}{2}$ hours. At autopsy there was intraventricular cerebral haemorrhage with spread into the subarachnoid space, and the lungs did not appear aerated.

Microscopic examination shows the lungs to be immature, and the pattern of resorption collapse, of dilated terminal bronchioles and alveolar ducts, with collapsed distal air-spaces, is not seen. Hyaline membranes are numerous (Fig. 2), and are of open texture, being formed of strands and coarsely granular material. The strands show positive staining with P.T.A.H., while the granules stain faintly or not at all. The lymphatics contain cell-free fibrin (Fig. 3), seen as a network of regular smooth-sided branching strands which stain deeply with P.T.A.H.; this network has shrunk during processing and now fills only part of the vessel. A few small groups of amniotic squames are present, but there is no haemorrhage or inflammation; polymorphonuclear 


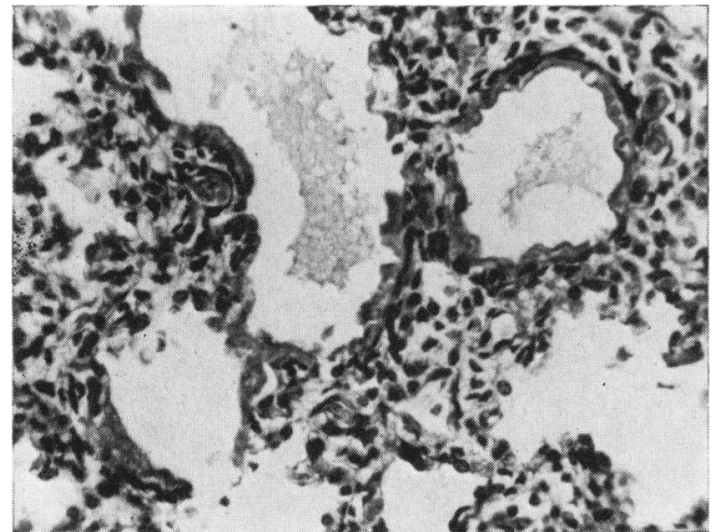

Fig. 2.-Case 1: thin hyaline membranes of open texture are found throughout both lungs. (Haematoxylin and eosin $\times 205$.)

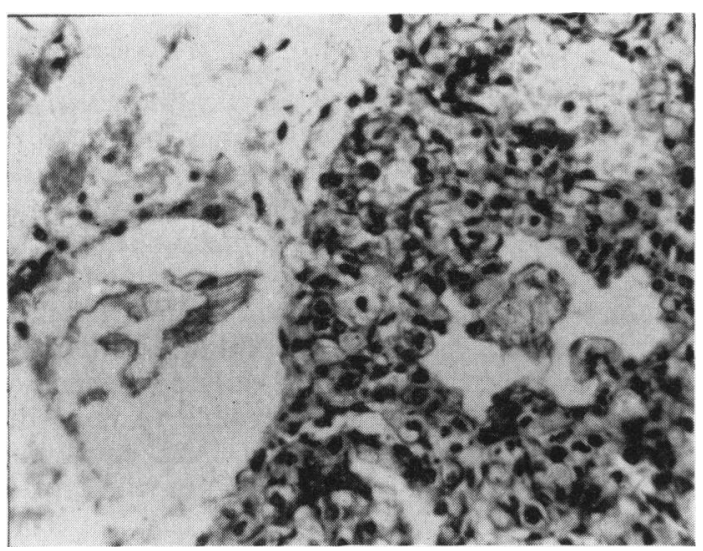

FIG. 3.-Case 1: both the pleural lymphatic (left) and the reripheral air space (right) contain strands of fibrin. (P.T.A.H. $\times 254$.)

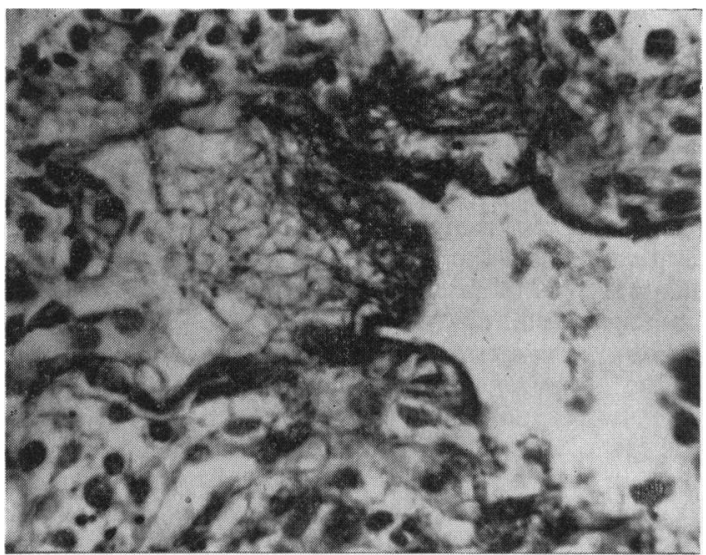

FIG. 4.-Case 1: a hyaline membrane in process of formation. Fluid containing strands of fibrin (left) is being compressed to form the denser membrane (right). (P.T.A.H. $\times$ 536.)

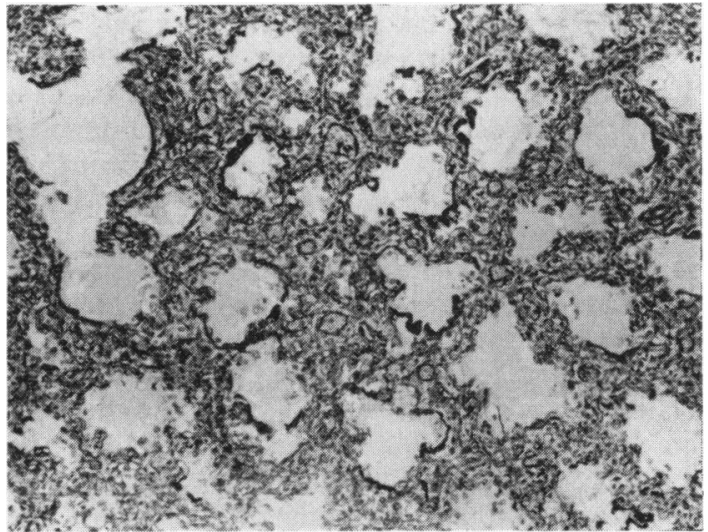

FIG. 5.-Case 2: the membranes are widespread, and in this area most stain through their whole thickness as fibrin. (P.T.A.H. $\times$ 68.)

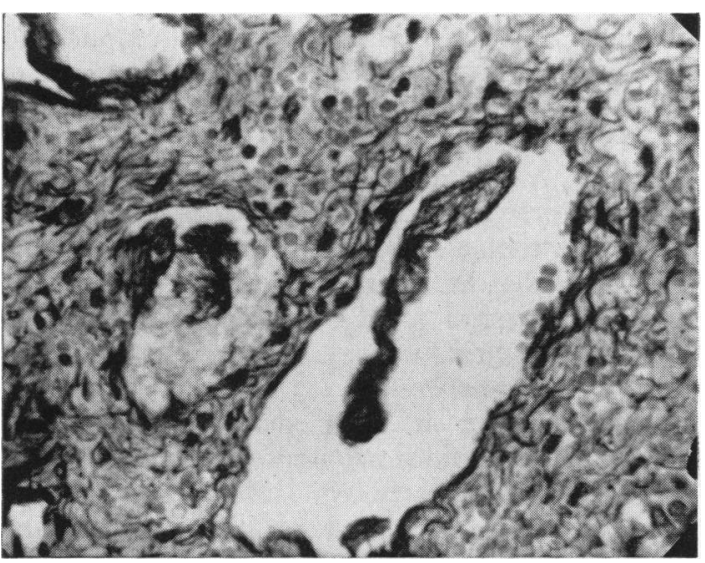

FIG. 6.-Case 2: fibrin strands within lymphatic vessels in an interlobular septum. (P.T.A.H. $\times$ 254.)

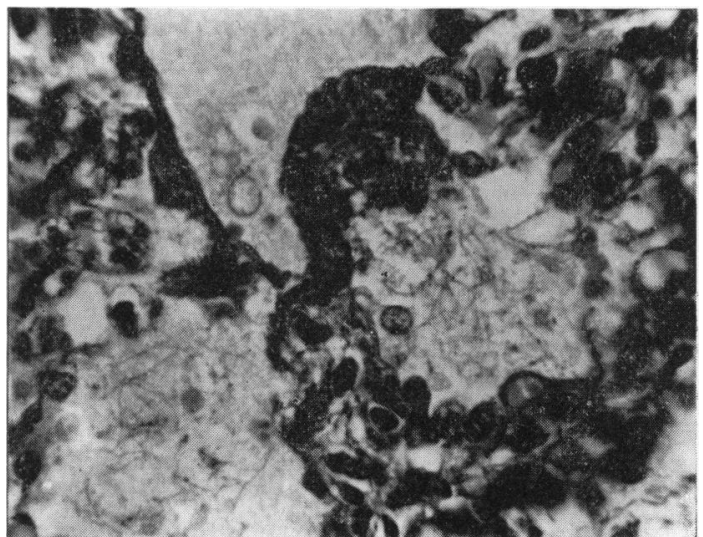

Fig. 7.-Case 2: compaction of fibrin-containing oedema fluid to form membranes. (P.T.A.H. $\times$ 536.) 
or other leucocytes are absent. Congestion is not marked, although the poor capillary development makes its recognition difficult.

Terminally the lungs were oedematous. The air spaces do not appear 'empty' as if they had contained air, but are filled by a coagulum which forms a fine irregular network, staining faintly with eosin and yellow or red with P.T.A.H. In places, however, the coagulum has a special character, containing narrow regular strands which are structurally and tinctorially identical with those of the fibrin in the lymphatics. This 'fibrinous oedema' is seen only in the most distal air spaces; at its interface with the simple oedema fluid it is condensed to form either more definite deeply stained strands or material identical with that forming the hyaline membranes (Fig. 4). These condensations are in places continuous with the formed hyaline membranes, although most of the latter have lost their specific staining.

The appearances suggest that the hyaline membranes have been formed by the partial absorption of fibrinous oedema fluid, with condensation at an interface which, although terminally with reaccumulated oedema fluid, was before that with air.

Case 2. Labour started spontaneously at 30 weeks in an 18-year-old primigravid patient; its course was normal, leading to the spontaneous vertex delivery of a $1.27 \mathrm{~kg}$. boy, who cried well soon after birth. At $4 \frac{1}{2}$ hours he was apnoeic for a few minutes, and after this was cyanosed; death occurred during a second apnoeic attack at 7 hours. At autopsy the lungs were airless and relatively heavy (right, 20 g., left, $16 \mathrm{~g}$.; heart, $10 \mathrm{~g}$.), and there was a little haemorrhage between the leaflets of the tentorium cerebelli, without tearing or subdural haemorrhage.

The lungs are more mature than those of the first case; peripherally there is less mesenchyme and, while the lining of the air spaces still has moderate numbers of cuboidal cells, capillaries are more numerous. The terminal bronchioles and the alveolar ducts are distended, possibly by attempted artificial respiration; the more distal air spaces are not, although the full picture of resorption collapse is not seen. Hyaline membranes are widespread (Fig. 5); in texture some resemble those seen in the first case, consisting of strands set among coarse granules, but many are formed throughout their width of thicker strands which stain with P.T.A.H. Polymorphs are seen beneath some but not all of the membranes, and there are small subpleural and interstitial petechial haemorrhages. The lymphatics in this case also contain fibrin (Fig. 6).

Otherwise, as in the first case, there is proximal to the membranes simple oedema fluid, and distal to them fluid that contains obvious strands of fibrin. These are compacted at the surface to form thicker strands which are in many places continuous with the fully-formed hyaline membranes (Fig. 7).

Case 3. A 33-year-old woman had had three abortions but no living children; when first examined in her fourth pregnancy she was found to be hypertensive.

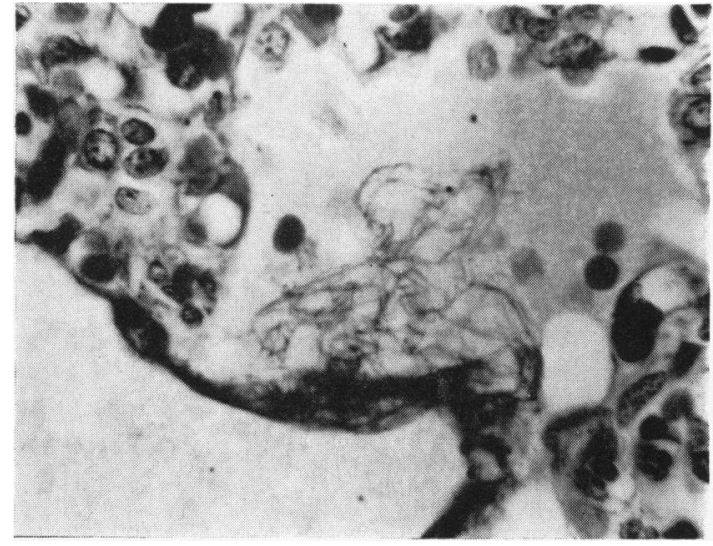

FIG. 8.-Case 3: a skein of delicate fibrin strands is seen beneath a membrane. (P.T.A.H. $\times 620$.)

At 29 weeks she had a small vaginal haemorrhage followed by the spontaneous onset of labour, with vertex delivery of a female child, who weighed $0.96 \mathrm{~kg}$. The child was cyanosed after delivery but gasped soon, and regular respiration commenced at 2 minutes. Respiration was from the first grunting, with inspiratory? rib recession, and remained so until the child died at $10 \frac{1}{2}$ hours. At autopsy the lungs were deep purple and unaerated (right $14 \mathrm{~g}$., left $12 \mathrm{~g}$.; heart $8 \mathrm{~g}$.); there was haemorrhage into both lateral ventricles with extension into the subarachnoid space.

Although the birth weight was less, the maturity of the lungs appears little different from that of the preceding case: capillary development is good with little mesenchyme between the peripheral air spaces and few cuboidal cells in their lining epithelium. Hyaline membranes are again widespread, some being of open texture and others being more compact, without differential staining. There is much fibrinous oedema, the strands being rather coarser than those seen in the first two cases. In many parts surface compaction is seen (Fig. 8), in some with the inclusion of amniotic squames or erythrocytes. Fibrin is not found in the lymphatics. The lungs are congested and oedematous, and small numbers of polymorphs are seen both in the fibrinous and the proximal oedema fluid.

Case 4. A 32-year-old primigravid patient was delivered by lower segment caesarean section, having had an eclamptic fit. She had been given large doses of sedative drugs. The child, birth weight $0.91 \mathrm{~kg}$., gasped soon after delivery but, while irregular respirations continued from this time, she remained cyanosed. At $22 \frac{1}{2}$ hours death occurred after a sudden apnoeic attack. At autopsy the lungs were dull purple and airless (right $12 \mathrm{~g}$., left $12 \mathrm{~g}$.; heart $9 \mathrm{~g}$.); there were no other important findings.

This case is of interest in that, although the age is greater than that of the preceding three, the histological features are similar (Figs. 9 and 10). Again, hyaline membranes are widespread, being mostly of uniform 


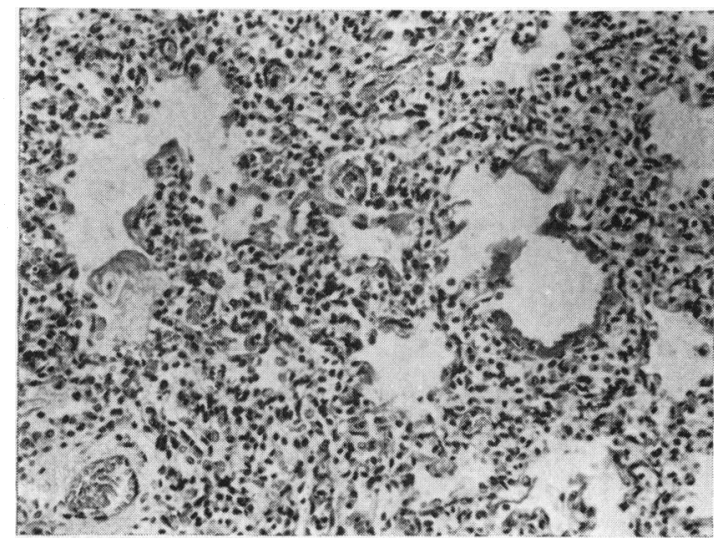

Fig. 9.-Case 4: the illustration shows both a complete hyaline membrane (right) and one in process of formation (left). A nucleated squame is included in the latter. (Haematoxylin and eosin $\times 124$.)

pattern, few appearing compact, and distally there is fibrinous oedema with compaction at its proximal surface. The lung is not obviously congested, the lymphatics do not contain fibrin, and polymorphs are rare.

Comment. These babies died with incompletely formed hyaline membranes which can be seen to arise from material identifiable, when its staining and structure are taken together, as fibrin. It seems unlikely that the fibrin is lost from capillaries damaged by membranes previously formed above them; in many places the membranes on the surface of the pockets of fibrin-containing oedema fluid are incomplete or even absent; continuity is often seen between strands in the underlying oedema and the membranes on its surface or at its margins. The observations recorded suggest that membranes arise by the condensation of this oedema fluid, and that the fibrin is their essential component; other materials are often present, but these are merely

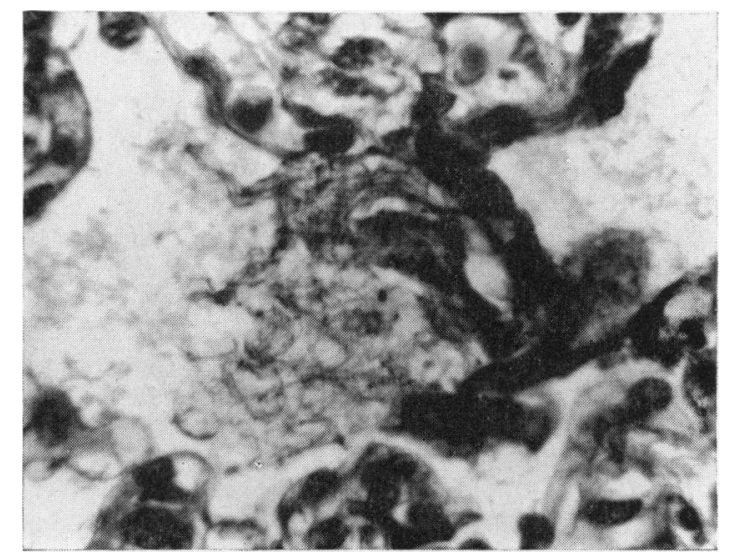

FIG. 10.-Case 4: high power view of a serial section of the partlyformed membrane seen in Fig. 9. (P.T.A.H. $\times$ 674.)

included within or absorbed onto a fibrinous matrix.

The formation of fibrinous fluid or gel must depend on an increase in permeability in the local capillaries which is great enough to allow the escape not only of serum proteins of low molecular weight, but of fibrinogen; further, there must be, first, a degree of recovery of capillary function great enough to allow the reabsorption of much of the fluid and soluble proteins and, second, aeration of the lung great enough to cause compression of the residual insoluble fibrin against the wall of the air space that contains it. This suggests that the formation of membranes is not a continuous process, but that it is of limited duration or is episodic, and further support is given to this conclusion by the occurrence of membranes which appear when early of 'open' and when late of 'compact' texture (Fig. 11). This is discussed further below.

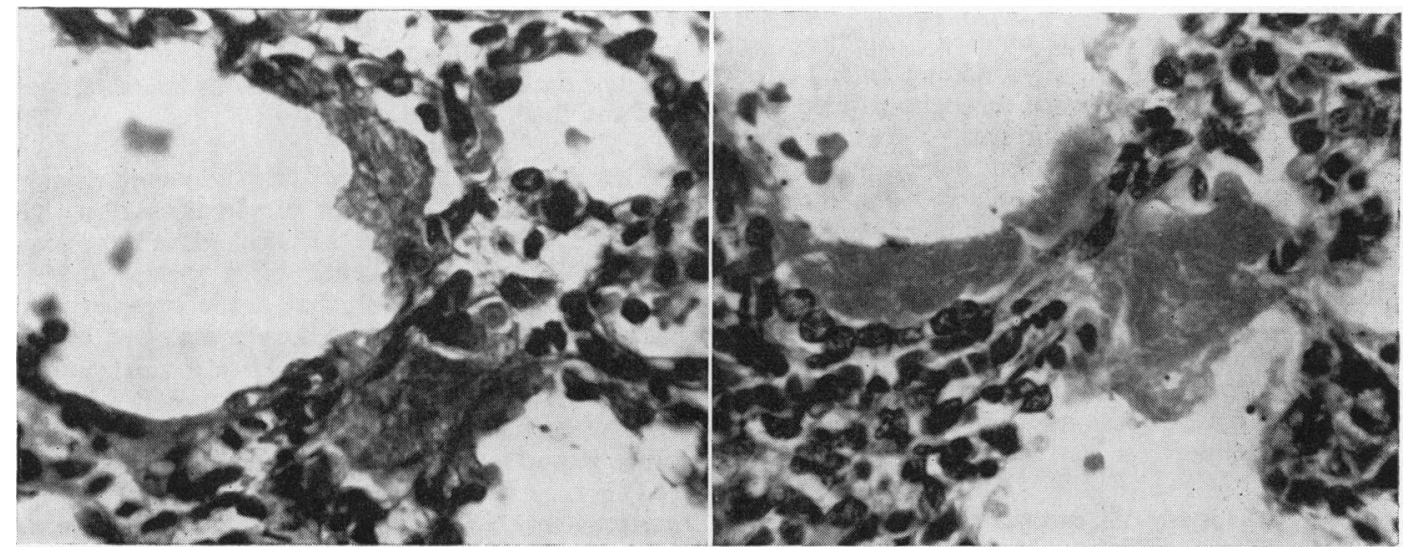

Fig. 11.-Hyaline membranes of contrasting texture. That from a $3 \frac{1}{2}$-hour-old baby (left) is open and strandlike, while that from a 19 -hourold baby (right) is more compact. (Haematoxylin and eosin, both approx. $\times 510$.) 


\section{The Cellular Reaction to Hyaline Membranes}

Small numbers of polymorphonuclear leucocytes are sometimes seen in the early stage of membrane formation, and pneumonia is a common late complication; the membranes themselves do not appear to excite a polymorph reaction, and in many cases dying at the end of the first or the beginning of the second day there is no leucocyte response of any type, although they have been present for many hours. In other cases, however, which survive for longer periods, active macrophages can be seen around the membranes; the two youngest babies with this change died at 33 hours. Examples of this reaction, and of further changes in babies of greater age, are presented below.

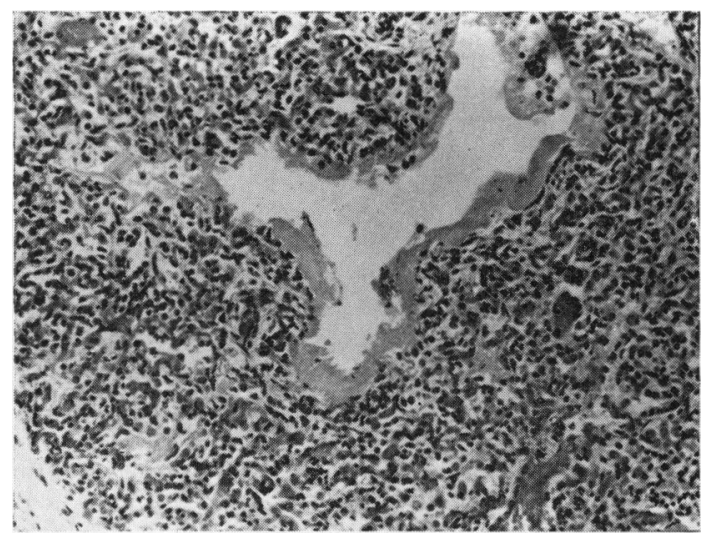

FIG. 12.-Case 5: hyaline membrane and 'resorption collapse', the tissue between the space containing the membrane and the pleura (lower left) appears solid. (Haematoxylin and eosin $\times 96$.)

Case 5. A 30-year-old multiparous patient had a small antepartum haemorrhage at 34 weeks and was found to have a marginal placenta praevia. Labour commenced spontaneously, leading to the precipitate vertex delivery of a $2.40 \mathrm{~kg}$. girl. At birth the baby was pale and limp, gasping respirations being established after a delay of seven to eight minutes; thereafter there was lower thoracic retraction, and she was cyanosed when not given oxygen. Respiration continued to be irregular, with periods of apnoea; a few hours before death, which occurred at 33 hours, venous congestion, enlargement of the liver and a cardiac murmur were noted. At autopsy the lungs were heavy, were uniformly dull red purple and airless (right $31 \mathrm{~g}$., left $27 \mathrm{~g}$.; heart $20 \mathrm{~g}$.); the heart appeared normal, and there were no other findings of importance.

On microscopical examination, the lungs are well developed and show the pattern of complete 'resorption' collapse; the terminal bronchioles and succeeding passages are dilated, but distal to these collapse is complete and the tissue appears solid (Fig. 12). Hyaline membranes are widespread; in general they are compact, without differential staining, although two contain pyknotic nuclei or nuclear debris. There are a few foci of recent inflammatory exudate proximal to the membranes, although these are not numerous. Macrophages are seen on the surface of, or within, some of the hyaline membranes (Fig. 13).

Case 6. Appendectomy was performed on a 31-yearold primigravid patient, 30 weeks pregnant. Labour commenced next day and was precipitate; the baby, a girl, was born in the surgical ward. On admission to the nursery she weighed $1.58 \mathrm{~kg}$. and was cyanosed with retraction of the lower thoracic wall. On the following day she had a number of cyanotic attacks, and the thoracic retraction increased. Later there were periods of

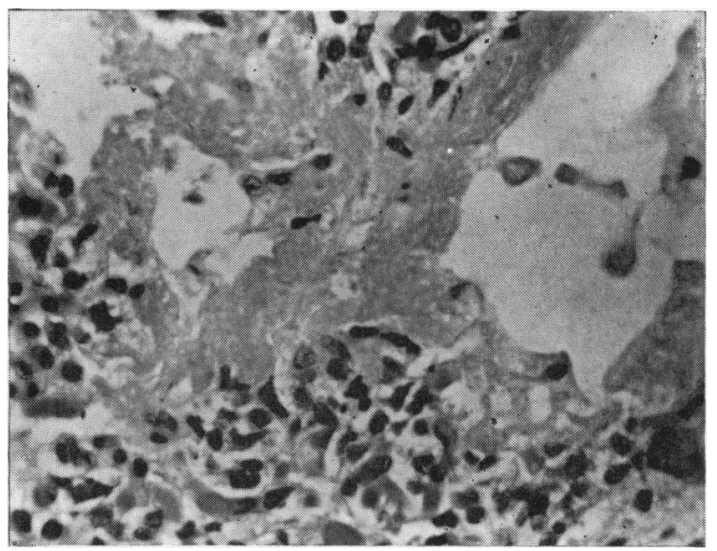

Fig. 13.-Case 5: Early macrophage response at 33 hours. (Haematoxylin and eosin $\times 254$.)

apnoea, one at 48 hours, and another just before death at 53 hours. At autopsy the lungs were heavy (right $29 \mathrm{~g}$., left $18 \mathrm{~g}$.; heart $12 \mathrm{~g}$.) and were 'atelectatic' with a narrow rim of aeration at the bases; a small haemorrhage was found between the folds of the falx, but there were no other findings of note.

On microscopical examination, the lungs appear mature and show the pattern of resorption collapse. Hyaline membranes are widespread, similar eosinophil material being seen in some of the cleft-like collapsed air spaces. The membranes are compact and give no differential staining with P.T.A.H. Distally are a few amniotic squames which have not excited a cellular response, although large active macrophages are closely applied to many of the membranes (Fig. 14). Some membranes have separated from the wall of the air spaces, and some are fragmented (Fig. 15), rounded masses of 'hyaline' material surrounded by macrophages lying free on the lumina; the epithelial lining cells deep to the membranes are often prominent, with deeply staining flattened nuclei which contrast with those of the macrophages. These features suggest that during life 


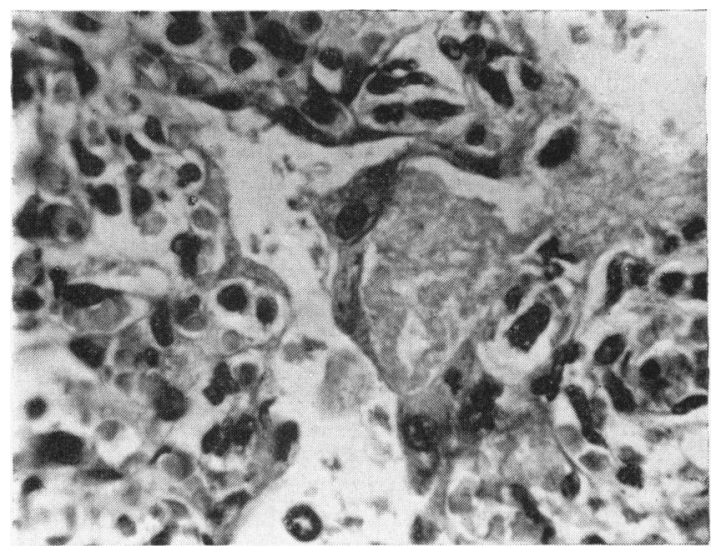

FIG. 14.-Case 6: later macrophage response at 53 hours. (Haematoxylin and eosin $\times 510$.)

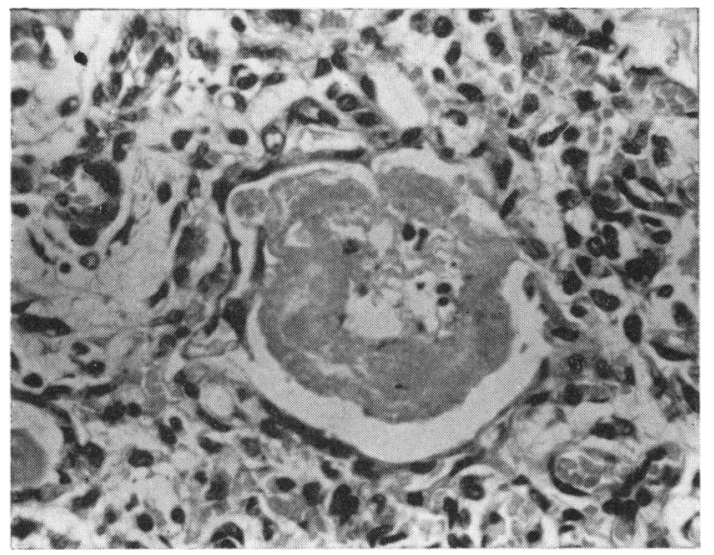

FIG. 15.-Case 6: the membrane is detached from the wall of the air space, whose lining cells are prominent, and is becoming fragmented. (Haematoxylin and eosin $\times 510$.)

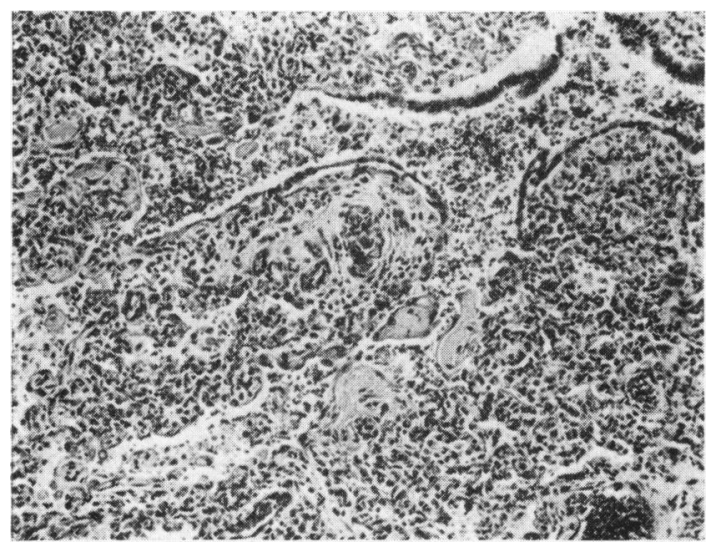

Fig. 16.-Case 7: the membrane is broken up, and macrophages surround small masses, some of which lie free in the air spaces. The epithelial lining cells are prominent. (Haematoxylin and eosin $\times$ 96.) the material composing the membranes was becoming detached and removed.

Case 7. A 30-year-old woman had had five children, one dying of haemolytic disease of the newborn. In her sixth pregnancy labour was induced at 36 weeks with a pitocin drip, and was followed by the normal vertex delivery of a $2.45 \mathrm{~kg}$. boy. He cried soon after birth, although rib recession was noted. He was jaundiced, the Coombs' test being positive, and the cord blood haemoglobin $84 \%$, and an exchange transfusion of $335 \mathrm{ml}$. was performed, starting at 2 hours. Rib recession persisted, however, and there was an apnoeic attack at 28 hours. On the third day kernikterus was diagnosed. Repeated apnoeic attacks followed in the succeeding days, and the baby died at the age of 6 days. At autopsy the lungs were dull purple, with areas of haemorrhage (right $32 \mathrm{~g}$., left $28 \mathrm{~g}$.; heart $24 \mathrm{~g}$.) ; there was well-marked kernikterus, although the liver $(110 \mathrm{~g}$.) and spleen $(10 \mathrm{~g}$.) were of normal size.

Recent diffuse haemorrhage into the air spaces with some fresh pneumonia makes histological interpretation difficult. In the lungs, which are mature, there is widespread hyaline membrane formation, and the membranes are surrounded by active macrophages, by which they are often split into small masses; similarly sized masses of 'hyaline' material surrounded by macrophages lie free in the air spaces (Fig. 16). In this case there is more epithelial activity; flattened cells can be seen below the membranes and sometimes upon their free surfaces, and in some places the columnar lining cells of the bronchioles form prominent groups, with hyperplastic nuclei which vary in size and show occasional mitotic figures.

Comment. These cases show that hyaline membranes in babies who survive long enough may be broken up and removed; this appears to be largely the result of macrophage activity, although it is possible that fibrinolysins may also play a part. In the last case epithelial cells lie on the surface of some of the membranes and these may have been in process of incorporation into the wall beneath; organization with the production of granulation or fibrous tissue was not seen, although the period of survival was probably too short for this. No case was found older than Case 7.

\section{Stages of Hyaline Membrane Formation}

The recognition of the early stage of membrane formation, with the presence of membranes of 'open' and 'compact' texture and, in late cases, of a cellular reaction, suggest criteria by which lungs with hyaline membranes may be classified into four stages. The earliest stage (Stage A) includes all those cases that show fibrinous oedema in the peripheral air spaces, whether evidence of compaction to form membranes is present or not; it seems 


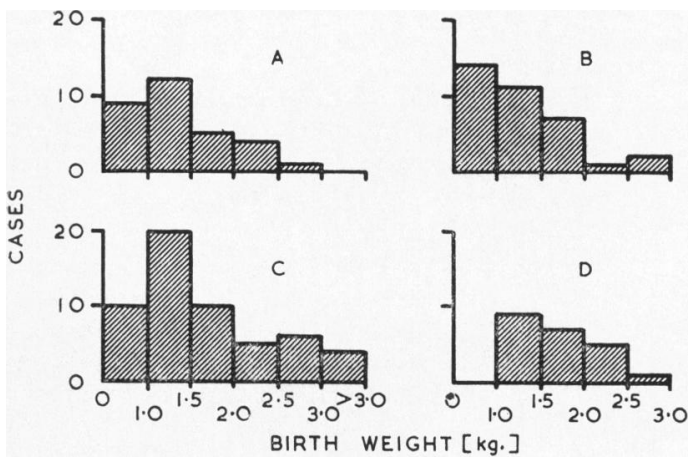

Fig. 17.-The distribution by birth weight of 143 babies with pulmonary hyaline membranes. Stages A-D.

justifiable to extend this group to include also the small number whose membranes appear to arise from granular material in which strands cannot be seen, either because the fibrin is present as a submicroscopic gel or because it has been removed by post-mortem fibrinolysis. The last stage (Stage D) includes all cases in which there is cellular reaction. Intermediate cases can be divided into an earlier group (Stage B) in which the membranes are open, and a later in which they are predominantly 'compact' (Stage C).

There may well be a yet earlier stage, in which fibrinous oedema fills the lung and drains through the lymphatics, without membrane formation. I have not yet seen a case which might be of this type in which pneumonia could be excluded with certainty; in the presence of even small numbers of leucocytes such a 'premembranous' stage would be impossible to distinguish from the earliest stage in an inflammatory reaction. It is possible that few babies die in this stage; that the loss of fibrin or fluid is greatest during recovery from an anoxic crisis would be in accordance with the hypothesis presented below.

Details of the 143 cases examined, classified in this way, are presented in the histograms (Figs. 17 and 18).

It has often been stated that babies with hyaline membranes pass through a period of relative wellbeing before the onset of respiratory distress, and an attempt was made in the present study to find such a turning point in the clinical history and to relate it to the histological findings. This did not prove possible, for in almost all cases respiratory distress began at birth and was continuous. However, from the data collected the duration of the various stages can be approximately established.

The youngest babies in Stage A had lived a little less than three hours; the minimum time needed for

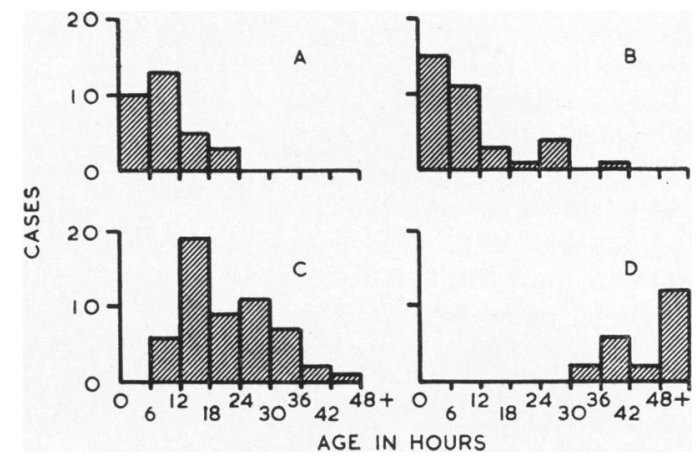

FIG. 18.-The distribution by age at death of 143 babies with pulmonary hyaline membranes. Stages A-D.

the escape of fibrinous fluid and its incomplete reabsorption appears to be between two and three hours. Although membrane formation has been reported in babies living less than this time, it is probable that at least some of these are the result of intrapartum pneumonia (Wade-Evans, 1961a). The process leading to the formation of membranes does not, however, always start soon after birth, but may be delayed until the end of the first day, as is seen in Case 4.

Most of the membranes found in babies who have lived for more than 12 hours are compact, although in a few who die at a little over 6 hours infrequent membranes of this type are seen; this period is probably the minimum needed for the formation of compact membranes. Such membranes persist without further alteration in babies surviving until the end of the second day, but beyond this all show surrounding macrophages. The two youngest babies in Stage D lived 33 hours; although it is not possible to be certain when the formation of their membranes began, it is likely that this also is the earliest time at which the cellular reaction commences.

\section{DISCUSSION}

It was concluded above that the pulmonary hyaline membranes seen in newborn babies are produced by partial reabsorption of fibrin-containing oedema fluid, with compression of the remaining insoluble fibrin, and that the preceding change is an increase in capillary permeability. The conditions under which this capillary lesion occurs may be summarized as follows: the change is greater than that involved in simple oedema formation, as it allows the escape of fibrinogen as well as of serum proteins of lower molecular weight; it is transient, as it is followed by some degree of recovery; it occurs widely throughout the lung, and is on the whole of uniform distribution. Further, it occurs 
in babies who, whatever causes their death, suffer from severe respiratory distress; it is confined almost entirely to the premature, with the exception of babies whose mothers have diabetes mellitus; and in the premature the incidence of membrane formation, if not the amount of 'hyaline' material present, increases with the degree of immaturity (Wade-Evans, 1960). Any hypothesis of membrane formation must account for these conditions.

There is, unfortunately, little direct evidence on the nature of the capillary alteration, although it is likely, as others have assumed, that it is the result of anoxic damage; the transience of the change argues against its being due to any inherent defect, such as maldevelopment or immaturity of the vessels, and against its being the result of any persistent or progressive change in the milieu interrieur; while it is of the degree found in inflammation, there is no evidence of a causative inflammatory reaction here, as all cases in which the membranes were thought to be due to pneumonia have been excluded (Wade-Evans, 1961a).

It seems paradoxical that the lung itself should be selectively damaged by anoxia, and this can happen only in certain circumstances. Drinker (1945) has pointed out that the pulmonary capillaries are unusual in that they normally receive oxygen from the inspired air, depending upon the circulation only when denied this direct access; it is a corollary of his view that anoxic damage must depend on a disturbance of both local aeration and circulation. That a circulatory change might contribute to hyaline membrane formation has previously been suggested by several workers. Thus Lendrum (1955) thought that such a disorder might occur in several stages; overloading of the pulmonary circulation, with loss of fluid from its capillaries, could occur early in independent life when the ductus arteriosus is completely closed, the flow through it being from left to right; this could cause anoxia, with the process accentuated by left ventricular failure; at such a time the better developed right ventricle might function more effectively than the left, causing reversal of the flow through the ductus with resorption of oedema fluid to leave insoluble protein material. In contrast, Polaček (1956) put forward a simpler hypothesis that closure of the ductus arteriosus would lead directly to overloading of the pulmonary circulation, with oedema, anoxia and increasing capillary damage. The occurrence of an increase in the number of thrombi in the hepatic sinusoids in babies with hyaline membranes has previously been presented as evidence in favour of an associated circulatory disturbance (Wade-Evans, 1961b).
It remains difficult to reconcile widespread anoxic damage to the lung with the supply to the rest of the body of oxygen sufficient for survival; unless the oxygen present at the beginning of an anoxic crisis is merely redistributed, an adequate amount must be absorbed from tissue of other than the lung, or at least other than the respiratory part of the lung. While small amounts may be absorbed through the skin or mucosae of other organs, it seems more likely that use is made of the greater area for respiratory exchange provided by the respiratory part of the lung; this is ventilated even during the disorderly respiration observed in babies with hyaline membranes. Indeed, the paradox of anoxic injury to the lung may be resolved if it is the vessels of the respiratory part rather than those of the conducting part that are damaged; that is, if a contributory disturbance of circulation involves a reduction in the pulmonary blood flow rather than the systemic.

If the hyaline membranes of the baby are compared with those that are sometimes formed from fibrinous oedema during circulatory disorders in older patients, for example, in severe left ventricular failure, the two are seen to differ in their distribution. In the adult the membranes are less widespread and, characteristically, are confined to the central part of the lung, whereas in the baby they are found more widely, extending into the subpleural as well as the more central lobules. Although in the adult the lesions are caused by heart failure, their distribution is probably determined by the structure or reaction of the vessels of the lung itself; the widespread and more regular distribution in the baby suggests that any causative circulatory change involves the lung as a whole, and possibly occurs outside it. Attention is thus drawn to factors outside the lung that influence the balance of the pulmonary and systemic circulations in the newborn, and in particular to the ductus arteriosus.

Much that has been learnt recently of the circulatory changes at birth, and of their modification by prematurity or anoxia, is of relevance to this problem. There is a period after birth during which the ductus arteriosus is constricted but not closed, blood flowing through it from left to right in a direction opposite to that of the foetus (Dawes, 1958); this has been shown most conclusively in the lamb, but is almost certainly also true of the baby. It is in this period that hyaline membrane formation occurs. Evidence from studies of oxygen saturation (Eldridge and Hultgren, 1955) and cardiac catheterization (James and Rowe, 1957) suggests that at this time anoxia, such as is produced by breathing $10 \%$ oxygen, causes a fall in systemic and a rise in 
pulmonary arterial pressure; the changes are sometimes so great that the direction of flow in the ductus must be reversed. Rudolph, Drorbaugh, Auld, Rudolph, Nadas, Smith and Hubbell (1961) found that in babies suffering severe respiratory distress the pulmonary arterial pressure was higher than normal, and that the ductus arteriosus was widely patent; in a small group found at autopsy to have hyaline membranes, the flow at the time of catheterization was from left to right in most, although the babies were at that time breathing $100 \%$ oxygen and were presumably in good general condition; in one who had a lower arterial oxygen saturation a right-to-left shunt was demonstrated.

Furthermore, a number of differences between the premature and mature have been revealed; in the premature the bruit ascribed to ductus flow is heard for a longer time (Burnard, 1959); it is likely in the baby as in the lamb that the decrease in pulmonary vascular resistance that follows ventilation is less in the premature; not only is the systemic systolic blood pressure less in babies than in adults, but its level is lower the lower the birth weight (Holland and Young, 1956; Young and Holland, 1958).

The hypothesis that follows is put forward in an attempt to explain the conditions of membrane formation outlined above in the light of this knowledge of the haemodynamics of respiratory distress in the newborn. Hyaline membranes are produced from fibrin-containing oedema fluid and are the consequences of a temporary increase in capillary permeability in the respiratory part of the lung. This is caused by severe anoxia, in part because there is a reduction in direct aeration and in part because there is an alteration in the pulmonary circulation. During an anoxic crisis blood pressure rises in the pulmonary arteries and falls in the aorta, the changes being great enough to allow a reversal of flow through the ductus. Blood is thus shunted from right to left, as in the foetus, and pulmonary blood flow is reduced, denying the respiratory part of the lung its share of the available oxygen, so that its capillary bed suffers anoxic damage. With commencement or restoration of respiration, these changes are reversed and pulmonary blood flow increases, although escape of fluid and proteins may at first continue, and may even be at its greatest at this time. If respiration is maintained, capillary function recovers completely with removal of fluid, leaving the insoluble fibrin to be compressed by the inflowing air.

Membrane formation is seen predominantly in the premature for two main reasons; first, the conditions of the circulation in the premature predispose to this reaction; second, and more important, the premature is more susceptible to severe anoxia because of the inadequacy of its pulmonary function, either in the smallest from simple immaturity of alveolar and capillary development, or in the slightly larger from the disability caused by abnormal aeration with differential expansion of the proximal part of the primary lobule to produce the so-called 'resorption collapse'.

If this is so, hyaline membranes must be considered a consequence of severe anoxia, and are denied the importance once given them as primary causes of pulmonary collapse or insufficiency; indeed, they probably contribute little if at all to such insufficiency. It is not appropriate to speak, as some have done, of a hyaline membrane 'disease' or 'syndrome', but rather of a hyaline membrane 'reaction' to anoxia, which may have many causes.

\section{SUMMARY}

The presence of fibrin within hyaline membranes, previously shown by the fluorescent antibody technique, is confirmed by studies using orthodox histological methods, and these suggest that fibrin is the essential component of the membranes. Stages in the formation of membranes from fibrincontaining oedema fluid and in their removal by macrophages are illustrated. It is concluded that hyaline membrane formation is due to a temporary increase in pulmonary capillary permeability; it is suggested that this capillary change is caused by anoxia, such as may occur when there is apnoea or delay in establishing respiration, and that it is in part the result of reduced aeration of the lung itself, and in part the result of a complicating disturbance of circulation in which the direction of flow through the ductus arteriosus returns to that found in the foetus, with a consequent reduction in pulmonary blood flow.

My thanks are due to Professor A. C. P. Campbell and to Professor W. I. C. Morris of the University of Manchester, in whose departments the work reported was performed, and to Dr. F. A. Langley for allowing me access to the autopsy material. Some of the observations were presented in a thesis accepted for the degree of Doctor of Medicine by the University of London.

\section{REFERENCES}

Aronson, Natalie (1961). Studies on hyaline membranes. Pediatrics, 27, 567.

van Breemen, V. L., Neustein, H. B. and Bruns, P. D. (1957). Pulmonary hyaline membranes studied with the electron microscope. Amer. J. Path., 33, 769.

Burnard, E. D. (1959). The cardiac murmur in relation to symptoms in the newborn. Brit. med. J., 1, 134.

Dawes, G. (1958). Changes in the circulation at birth and the effects of asphyxia. In Recent Advances in Paediatrics, ed. D. Gairdner, 2nd ed., p. 1. Churchill, London.

Drinker, C. K. (1945). Pulmonary Edema and Inflammation, p. 29. Harvard University Press, Cambridge, Mass. 
Eldridge, F. L. and Hultgren, H. N. (1955). The physiologic closure of the ductus arteriosus in newborn infant. J. clin. Invest., 34,987 .

Gitlin, D. and Craig, J. M. (1956). Nature of hyaline membrane in asphyxia of newborn. Pediatrics, 17, 64. - (1957). Variations in the staining characteristics of human fibrin. Amer. J. Path., 33, 267.

Hochheim, K. (1903). Ueber einige Befunde in den Lungen von Neugeborenen und die Beziehung der derselben zur Aspiration von Fruchtwasser. In Pathologisch-anatomische Arbeiten [Festschrift für Johannes Orth]. p.421. Hirschwald, Berlin

Holland, W. W. and Young, I. Maureen (1956). Neonatal blood pressure in relation to maturity, mode of delivery, and condition pressure in relation to maturity,

James, L. S. and Rowe, R. D. (1957). The pattern of response of pulmonary and systolic arterial pressures in newborn and older infants to short periods of hypoxia. J. Pediat., 51, 5

Lendrum, F. C. (1955). "Pulmonary hyaline membrane"; as a manifestation of heart failure in the newborn infant. ibid., 47, 149.
Lorand, L. (1950). Fibrin clots. Nature (Lond.), 166, 694.

Polacek, K. (1956). The pathogenesis of hyaline membrane disease. Etud. néo-natal., 5, 31.

Rudolph, A. M. Drorbaugh, J. E., Auld, P. A. M., Rudolph, A. J. Nadas, A. S., Smith, C. A. and Hubbell, J. P. (1961). Studies on the circulation in the neonatal period. The circulation in the respiratory distress syndrome. Pediatrics, 27, 551 .

Wade-Evans, T. (1960). The Formation of Hyaline Membranes in the Lung of the Newborn Baby, pp. 49 and 181. M.D. Thesis, University of London.

(1961a). Pulmonary hyaline membranes, aspiration and pneumonia. Arch. Dis. Childh., 36, 293.

(1961b). Thrombi in the hepatic sinusoids of the newborn and their relation to pulmonary hyaline membrane formation. ibid., 36, 286.

Young, I. Maureen and Holland, W. W. (1958). Some physiological responses of neonatal arterial blood pressure and pulse rate. Brit. med. J., 2, 276. 